\title{
Nonlocal amplification of intense vorticity in turbulent flows
}

\author{
Dhawal Buaria $\odot^{1,2, *}$ and Alain Pumir $\oplus^{3,2}$ \\ ${ }^{1}$ Tandon School of Engineering, New York University, New York, New York 11201, USA \\ ${ }^{2}$ Max Planck Institute for Dynamics and Self-Organization, Göttingen 37077, Germany \\ ${ }^{3}$ Laboratoire de Physique, Ecole Normale Supérieure de Lyon, Université de Lyon 1 and Centre National \\ de la Recherche Scientifique, 69007 Lyon, France
}

(Received 13 May 2021; accepted 19 October 2021; published 10 November 2021)

\begin{abstract}
The nonlinear and nonlocal coupling of vorticity and strain rate constitutes a major hindrance in understanding the self-amplification of velocity gradients in turbulent fluid flows. Utilizing highly resolved direct numerical simulations of isotropic turbulence in periodic domains of up to $12288^{3}$ grid points and Taylor-scale Reynolds number $R_{\lambda}$ in the range 140-1300, we investigate this nonlocality by decomposing the strain-rate tensor into local and nonlocal contributions obtained through Biot-Savart integration of vorticity in a sphere of radius $R$. We find that vorticity is predominantly amplified by the nonlocal strain coming beyond a characteristic scale size, which varies as a simple power law of vorticity magnitude. The underlying dynamics preferentially align vorticity with the most extensive eigenvector of nonlocal strain. The remaining local strain aligns vorticity with the intermediate eigenvector and does not contribute significantly to amplification; instead it surprisingly attenuates intense vorticity, leading to breakdown of the observed power law and ultimately also the scale invariance of vorticity amplification, with important implications for prevailing intermittency theories.
\end{abstract}

DOI: 10.1103/PhysRevResearch.3.L042020

Complex nonlinear physical systems are often characterized by formation of extreme events, which strongly deviate from Gaussianity, necessitating anomalous corrections to mean-field descriptions [1-3]. Fluid turbulence, described by the three-dimensional incompressible Navier-Stokes equations (INSE), is an emblematic example of such a system, where extreme events are associated with intermittent formation of large velocity gradients, organized into thin filaments of intense vortices [4-7]. The amplification of such intense gradients is readily described by the vortex-stretching mechanism, which expresses the nonlinear stretching of vorticity $\omega$, by the strain-rate tensor $S_{i j}$ in the INSE (written as the vorticity equation)

$$
\frac{D \omega_{i}}{D t}=\omega_{j} S_{i j}+v \nabla^{2} \omega_{i},
$$

where $v$ is the kinematic viscosity.

The canonical description based on angular momentum conservation dictates that as vortical filaments are stretched by strain, they become thinner and spin more quickly, enabling gradient amplification and simultaneously driving the energy cascade from large to small scales [8,9]. Though Eq. (1) is valid pointwise, this multiscale description can be analyzed

\footnotetext{
*dhawal.buaria@nyu.edu
}

Published by the American Physical Society under the terms of the Creative Commons Attribution 4.0 International license. Further distribution of this work must maintain attribution to the author(s) and the published article's title, journal citation, and DOI. Open access publication funded by the Max Planck Society. by realizing that vorticity and strain are related nonlocally via Biot-Savart integral over the entire flow domain:

$$
S_{i j}(\mathbf{x})=P V \int_{\mathbf{x}^{\prime}} \frac{3}{8 \pi}\left(\epsilon_{i k l} r_{j}+\epsilon_{j k l} r_{i}\right) \omega_{l}\left(\mathbf{x}^{\prime}\right) \frac{r_{k}}{r^{5}} d^{3} \mathbf{x}^{\prime},
$$

where $\mathbf{r}=\mathbf{x}-\mathbf{x}^{\prime}, r=|\mathbf{r}|$, and $\epsilon_{i j k}$ is the Levi-Civita symbol. This integral essentially couples all the scales, providing a direct means to understand the nonlocality of gradient amplification, without involving additional complexities such as the pressure field $[10,11]$. However, the integral in Eq. (2) is analytically intractable, leading to outstanding challenges in turbulence theory and also in establishing the regularity of INSE [12]. In this Letter, we investigate the nonlocality of vorticity self-amplification by tackling the Biot-Savart integral in Eq. (2) via direct numerical simulations (DNS) of INSE [6].

To analyze the nonlocality with regard to a scale size $R$, the integration domain in Eq. (2) is separated into a spherical neighborhood of radius $r \leqslant R$ and the remaining domain [13-15]:

$$
S_{i j}(\mathbf{x})=\underbrace{\int_{r>R}[\cdots] d^{3} \mathbf{x}^{\prime}}_{=S_{i j}^{\mathrm{NL}}(\mathbf{x}, R)}+\underbrace{\int_{r \leqslant R}[\cdots] d^{3} \mathbf{x}^{\prime}}_{=S_{i j}^{\mathrm{L}}(\mathbf{x}, R)},
$$

where $S_{i j}^{\mathrm{NL}}$ represents the nonlocal or background strain acting on the vorticity to stretch it and $S_{i j}^{\mathrm{L}}$ is the local strain induced in response to stretching. We utilize DNS to compute $S_{i j}^{\mathrm{L}, \mathrm{NL}}$ and investigate their interaction with vorticity for various $R$, allowing us to quantify the degree of nonlocality of vortex stretching and thereafter relate it to vortical structures in the flow. 

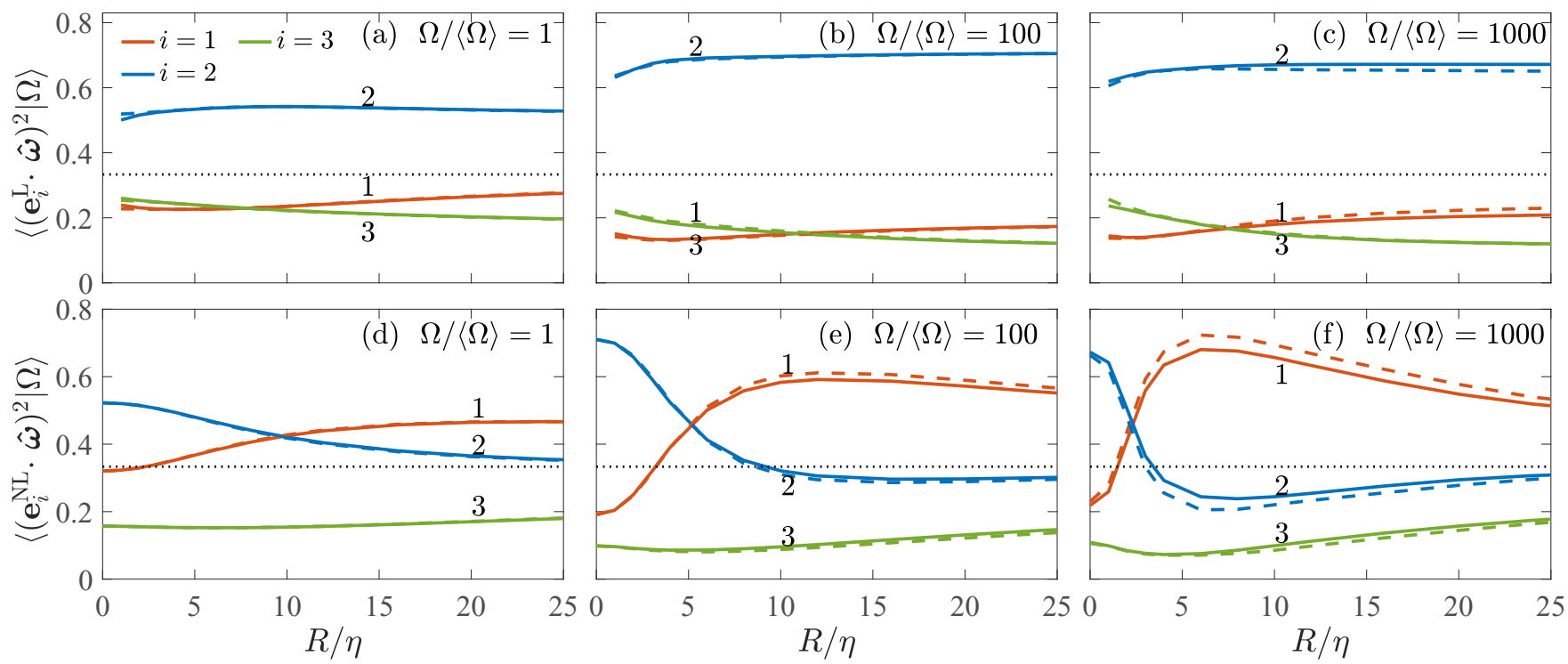

FIG. 1. Conditional second moment of the alignment cosines between vorticity and eigenvectors of the local (L) and nonlocal (NL) strain tensors at $R_{\lambda}=1300$ (solid lines) and 650 (dashed lines), and various conditioning values of enstrophy $\Omega$. The dotted line at $1 / 3$ in each panel corresponds to a uniform distribution of the cosines. Note that $S_{i j}^{\mathrm{L}}=0$ at $R=0$, with the alignments being undefined.

While computing $S_{i j}^{\mathrm{L}, \mathrm{NL}}$ through numerical integration is possible in DNS [14], it is prohibitively expensive at high Reynolds numbers [16]. Instead, as derived in our recent work [15], nonlocal (and local) strain can be efficiently computed for any $R$ by applying a transfer function to the total strain in Fourier space: $\hat{S}_{i j}^{\mathrm{NL}}(\mathbf{k}, R)=f(k R) \hat{S}_{i j}(\mathbf{k})$, with $f(k R)=3[\sin (k R)-k R \cos (k R)] /(k R)^{3}$, thus bypassing the direct evaluation of the Biot-Savart integral. This approach is used to analyze a large DNS database, generated using the canonical setup of forced stationary isotropic turbulence in a periodic domain [6], utilizing the highly accurate Fourier pseudospectral methods [17]. Special attention is given to maintain a grid resolution smaller than the Kolmogorov length scale $\eta$ to resolve the extreme events accurately [7]. The database corresponds to Taylor-scale Reynolds number $R_{\lambda}$ in the range 140-1300, on up to grids of $12288^{3}$ (for additional details, see Refs. [15,18-20]).

The efficacy of vortex stretching is controlled by the alignment between vorticity and strain rate and is commonly studied in the eigenframe of strain tensor, given by the eigenvalues $\lambda_{i}\left(\lambda_{1} \geqslant \lambda_{2} \geqslant \lambda_{3}\right)$ and the corresponding eigenvectors $\mathbf{e}_{i}$. Incompressibility imposes $\lambda_{1}+\lambda_{2}+\lambda_{3}=0$, giving $\lambda_{1}>$ 0 and $\lambda_{3}<0$. (The corresponding quantities for local and nonlocal strain are defined with superscripts $\mathrm{L}$ and NL, respectively.) It is well known that $\lambda_{2}$ is positive on average and vorticity preferentially aligns with the intermediate (second) eigenvector of the total strain rate [19,21-23]. This alignment is often regarded as anomalous, since an analogy with stretching of material lines suggests that vorticity should align with the first eigenvector of total strain, corresponding to the largest eigenvalue [24].

The earlier work of Ref. [14], based on direct evaluation of the Biot-Savart integral for a single value of $R=12 \eta$ at very low Reynolds number $R_{\lambda} \approx 100$, provides some evidence that vorticity preferentially aligns with the first eigenvector of the nonlocal strain (similar to stretching of material lines), whereas the anomalous alignment results from local dynamics. In the following, we provide a comprehensive investigation of the alignment properties, as a function of $R$ and over a drastically larger $R_{\lambda}$ range. In addition, we also condition on the enstrophy, $\Omega=\omega_{i} \omega_{i}$, to analyze generation of intense vorticity. To this end, we extract the second moment of directional cosines: $\left\langle\left(\mathbf{e}_{i}^{\mathrm{L}, \mathrm{NL}} \cdot \hat{\boldsymbol{\omega}}\right)^{2}\right\rangle$, whose averages are individually bounded between 0 and 1 (with $1 / 3$ corresponding to a uniform distribution), and additionally also add up to unity, i.e., $\sum_{i=1}^{3}\left(\mathbf{e}_{i}^{\mathrm{L}, \mathrm{NL}} \cdot \hat{\boldsymbol{\omega}}\right)^{2}=1[19]$.

The directional cosines are shown as a function of scalesize $R / \eta$ in Fig. 1, and conditioned on $\Omega /\langle\Omega\rangle$ to separate the extreme events. The alignments for $\mathbf{S}^{\mathrm{L}}$ are explored first in Figs. 1 (a) -1 (c), corresponding to $\Omega /\langle\Omega\rangle=1,100,1000$. We observe that for all $R / \eta$, vorticity preferentially aligns with second eigenvector of $\mathbf{S}^{\mathrm{L}}$, with a tendency to be orthogonal to first and third eigenvectors. The alignment properties become more pronounced as $\Omega$ increases. Overall, this result conforms to the picture of axisymmetric vortex tubes, where the velocity field is approximately two-dimensional, resulting in preferential alignment of vorticity with the second eigenvector of $\mathbf{S}^{\mathbf{L}}[13,25,26]$. Interestingly, vorticity is more orthogonal to the first eigenvector compared to the third for small $R$ $(\lesssim 10 \eta)$, with the difference becoming more pronounced for large $\Omega$ in Fig. 1(c) (we return to this behavior later). At large $R$, this trend is reversed, approaching the well-known result corresponding to total strain (as $\mathbf{S}^{\mathrm{L}}=\mathbf{S}$ for $R \rightarrow \infty$ ) [19,21].

The alignment of vorticity with $\mathbf{S}^{\mathrm{NL}}$ is shown next in Figs. 1(d)-1(f). The known alignment between vorticity and the intermediate eigenvector of $\mathbf{S}$ is recovered at $R=0$ (where $\left.\mathbf{S}^{\mathrm{NL}}=\mathbf{S}\right)$. However, as $R$ increases, a switch occurs and $\boldsymbol{\omega}$ preferentially aligns with the first eigenvector of $\mathbf{S}^{\mathrm{NL}}$, more strongly as $\Omega$ increases (while vorticity is always preferentially orthogonal to third eigenvector) [27]. These results clearly demonstrate that vortices are predominantly stretched by the nonlocal strain in a manner similar to passive material 


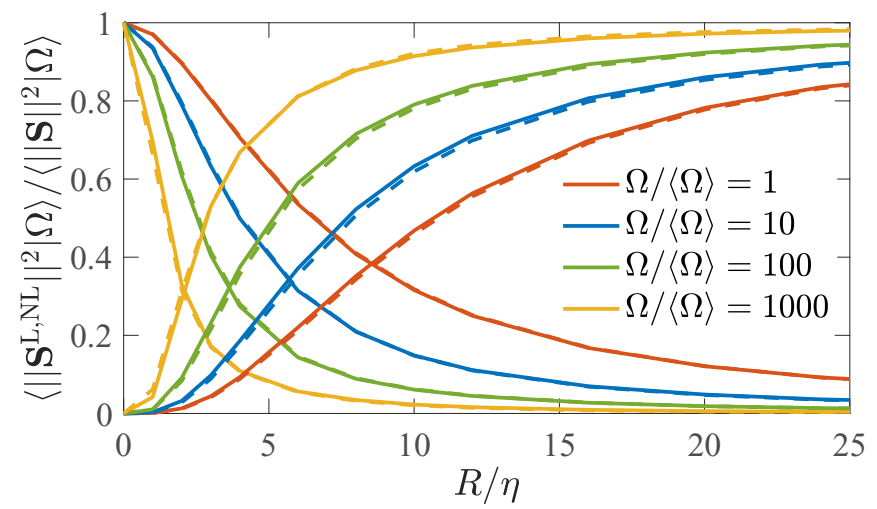

FIG. 2. Conditional expectation of the square-norm of local (L) and nonlocal (NL) strain tensor, normalized by the corresponding expectation of total strain, as a function of $R / \eta$, at $R_{\lambda}=1300$ (solid lines) and $R_{\lambda}=650$ (dashed lines). The curves for local strain start from zero at $R=0$.

lines, with vorticity preferentially aligned with the most extensive eigenvector. However, in the vicinity of these vortices, the (local) induced strain causes the alignment to switch from first to second eigenvector.

Figures 1(d)-1(f) show that the switching of alignment occurs at a distance $R_{c}^{a}=R_{c}^{a}(\Omega)$, which decreases with $\Omega$. This behavior also manifests itself when comparing the relative magnitudes of $\mathbf{S}^{\mathrm{L}, \mathrm{NL}}$. Figure 2 shows the $R$ dependence of the conditional expectation of the norm of $\mathbf{S}^{\mathrm{L}, \mathrm{NL}}$. They are normalized by the corresponding conditional expectation of total strain, which constrains the curves for $\mathbf{S}^{\mathrm{NL}}$ and $\mathbf{S}^{\mathrm{L}}$ at unity at $R=0$ and $\infty$ respectively. As $\Omega$ increases, the normalized magnitude of $\mathbf{S}^{\mathrm{L}}$ approaches unity at a smaller $R$, whereas that of $\mathbf{S}^{\mathrm{NL}}$ falls off toward zero in a similar fashion. This critical distance, say $R_{c}(\Omega)$, at which their relative magnitudes are equal, steadily decreases with $\Omega$, qualitatively consistent with the switching of alignment in Figs. 1(d)-1(f).

The results in Figs. 1 and 2 allow us to identify characteristic length scales, which demarcate the relative importance of local and nonlocal dynamics, and its dependence on $\Omega$. The analysis of Burgers vortices presented in Ref. [13] establishes that $R_{c}^{a}(\Omega) \simeq R_{c}(\Omega)$, and they physically identify the radii of vortex tubes in the flow [14]. A simple method to obtain the radius of a vortex tube is from a balance between viscosity $v$ and some effective strain $S$, giving $R=(v / S)^{1 / 2}$ [29]. Utilizing strain corresponding to mean field, i.e., $S \sim\langle\epsilon\rangle / \nu$, where $\langle\epsilon\rangle$ is the mean dissipation rate, results in the well-known expression for the Kolmogorov length scale $\eta=\left(v^{3} /\langle\epsilon\rangle\right)^{1 / 4}$. However, strain acting on intense vorticity grows with vorticity, given by the power law $[7,19]$

$$
\left\langle\|\mathbf{S}\|^{2} \mid \Omega\right\rangle \sim \Omega^{\gamma}, \quad 0<\gamma<1,
$$

where the exponent $\gamma$ weakly increases with $R_{\lambda}$, ostensibly approaching unity at $R_{\lambda} \rightarrow \infty$ [28]. Utilizing Eq. (4), and $\langle\epsilon\rangle=\nu\langle\Omega\rangle$ from statistical homogeneity, the radius of tubes $R^{*}$ can be written as a function of $\Omega$ :

$$
R^{*} / \eta \sim(\Omega /\langle\Omega\rangle)^{-\gamma / 4} .
$$

To test the result in Eq. (5), Fig. 3 shows the curves for $R_{c}^{a}(\Omega)$ (dashed lines) and $R_{c}(\Omega)$ (solid lines) extracted from

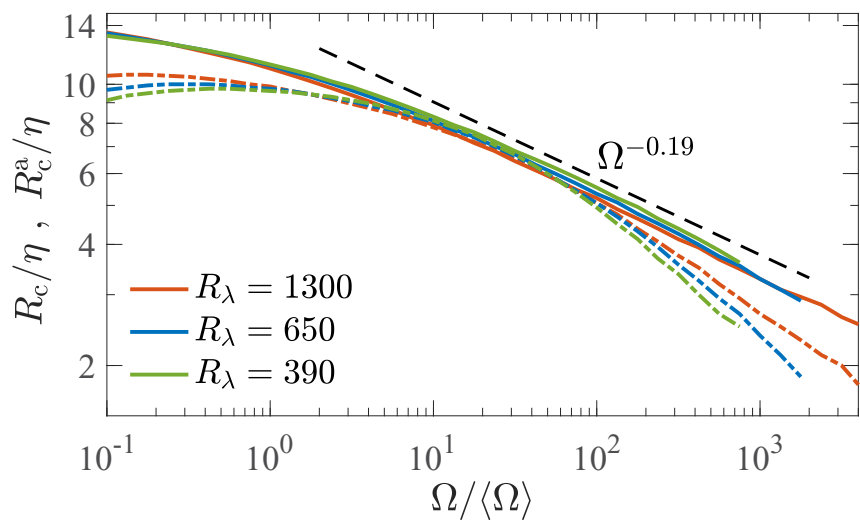

FIG. 3. The critical distances $R_{c} / \eta$ (solid lines) and $R_{c}^{a} / \eta$ (dashed lines), as a function of $\Omega$, respectively, corresponding to switching of alignment in Figs. 1(d)-1(f), and the distance obtained from Fig. 2 where magnitude of conditional local and nonlocal strain are equal. The black dotted line corresponds $\Omega^{-0.19}$, based on Eq. (5), with $\gamma=$ 0.76 for $R_{\lambda}=1300$ [19,28].

from Figs. 1(d)-1(f) and Fig. 2, respectively. First, we observe that both $R_{c}(\Omega)$ and $R_{c}^{a}(\Omega)$ are always comparable and follow the same trend for moderately intense vorticity, consistent with the power law predicted by Eq. (5) (represented by the black dashed line). For very intense events $(\Omega /\langle\Omega\rangle \gtrsim 100)$, $R_{c}(\Omega)$ is still consistent with the power law, but $R_{c}^{a}(\Omega)$ starts deviating. However, these deviations occur at slightly increasing values of $\Omega$ when $R_{\lambda}$ increases. We note that over the range of $R_{\lambda}$ (from 390 to 1300 ), the exponent $\gamma / 4$ only varies from 0.17 to 0.19 (respectively), and this small change in slope is also faintly visible for the curves corresponding to $R_{c}$. It is worth noting that such a dependence of vortex radius on $\Omega$ was not possible to detect in earlier studies at significantly lower $R_{\lambda}[5,14]$.

To analyze deviations of $R_{c}^{a}$ at large $\Omega$, we consider the enstrophy production term, $P_{\Omega}=\omega_{i} \omega_{j} S_{i j}$ which also represents the effective strain acting to amplify vorticity by factoring in the alignments. Similar to Eq. (3), we can also decompose $P_{\Omega}$ as $P_{\Omega}=P_{\Omega}^{\mathrm{L}}+P_{\Omega}^{\mathrm{NL}}$, where $P_{\Omega}^{\mathrm{L}, \mathrm{NL}}=\omega_{i} \omega_{j} S_{i j}^{\mathrm{L}, \mathrm{NL}}$. The conditional expectation of the nonlocal production $\left\langle P_{\Omega}^{\mathrm{NL}} \mid \Omega\right\rangle$, normalized by the total conditional production $\left\langle P_{\Omega} \mid \Omega\right\rangle$, is shown in Fig. 4.

For regions of moderately strong vorticity $(\Omega \lesssim 10\langle\Omega\rangle)$, the normalized production term $P_{\Omega}^{\mathrm{NL}}$ behaves qualitatively similar as nonlocal strain in Fig. 2; it starts at unity for $R=0$ and monotonically decreases to zero at $R \rightarrow \infty$. However, when conditioned on extreme values of $\Omega(\gtrsim 100\langle\Omega\rangle)$, the normalized $P_{\Omega}^{\mathrm{NL}}$ overshoots unity at small $R$, before decreasing more sharply at larger $R$. Since $P_{\Omega}^{\mathrm{L}} / P_{\Omega}=1-P_{\Omega}^{\mathrm{NL}} / P_{\Omega}$, this observation implies that local production is negative for small $R$, and thus counteracts vorticity amplification for large $\Omega$. This is in fact a manifestation of the self-attenuation mechanism recently identified in Ref. [15], which provides an inviscid mechanism to arrest vorticity growth and supports regularity of Navier-Stokes equations. Note that viscosity plays an implicit role, since stationarity imposes a conditional balance between net inviscid production and viscous destruction, so that the self-attenuation mechanism manifests 


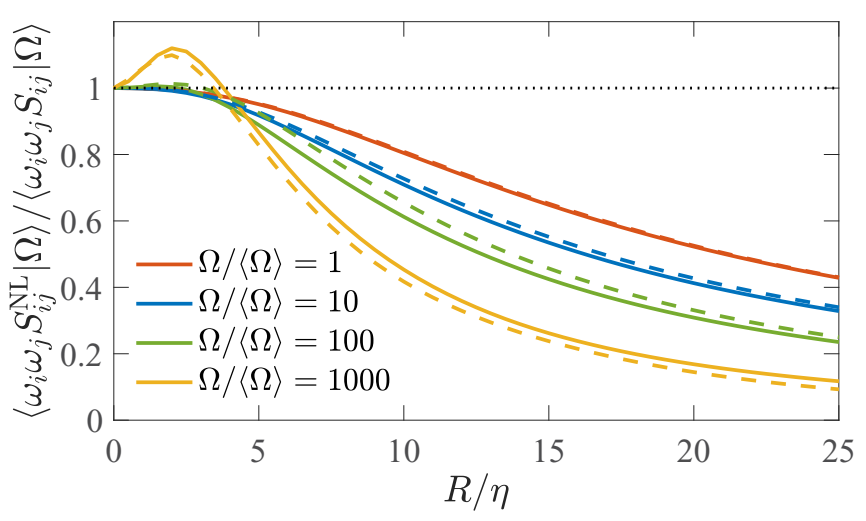

FIG. 4. Conditional expectation of the enstrophy production based on nonlocal strain, $\left\langle\omega_{i} \omega_{j} S_{i j}^{\mathrm{NL}} \mid \Omega\right\rangle$, normalized by the corresponding enstrophy production for total strain, as a function of $R / \eta$, at $R_{\lambda}=1300$ (solid lines) and 650 (dashed lines).

at increasing $\Omega$ values with $R_{\lambda}$ [15], in agreement with the deviations of $R_{c}^{a}(\Omega)$ in Fig. 3.

A breakdown of individual contributions from each eigenvalue for both $P_{\Omega}^{\mathrm{L}, \mathrm{NL}}$, normalized by the total production, is shown next in Fig. 5. Figures 5(a) and 5(b) show that the first eigenvalue of nonlocal strain provides most of the production, with the contributions from the second and third eigenvalues largely canceling each other, except at small $R$, where the second eigenvalue provides a small but significant contribution. The contributions to the local production in Figs. 5(c) and 5(d) show a very weak role of the intermediate eigenvalue for small $R$, despite the very strong alignment observed in Figs. 1(a)-1(c). Rather, the contributions from first and third eigenvalues are more prominent, with the third eigenvalue ultimately leading to overall negative local production at large $\Omega$ and small $R$ [which can also be traced to the slightly better alignment of vorticity with the third eigenvector instead of the first, also observed in Figs. 1(a)-1(c)]. These

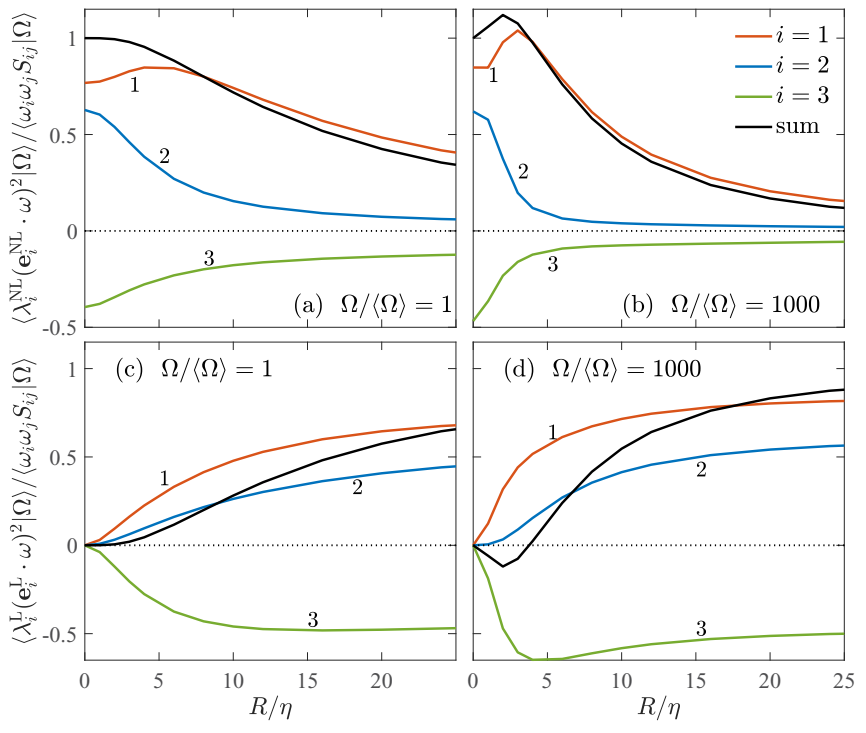

FIG. 5. The individual contributions from each eigenvalue to the nonlocal (NL) and local (L) enstrophy production terms, normalized by the production based on total strain, at $R_{\lambda}=1300$.

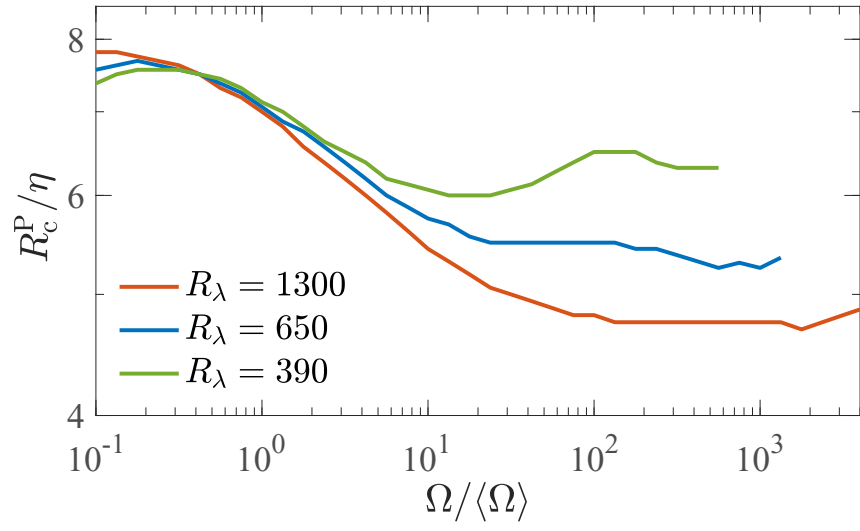

FIG. 6. The critical distance $R_{c}^{P} / \eta$ for which non-local enstrophy production accounts for $90 \%$ of total production (as derived from Fig. 4).

results highlight the nontrivial role of nonlinearity, going beyond a simple kinematic alignment switching as hypothesized earlier [13,25].

The results in Figs. 4 and 5 reiterate that vorticity is predominantly amplified nonlocally, analogous to linear dynamics of material line stretching, whereas the nonlinear effects are local and restricted to small distances but still play an important role. Since vorticity is amplified beyond a threshold, the local effects directly counteract further amplification, reflecting a fundamental change in the nature of extreme events. It marks a breakdown of scale invariance (self-similarity) of vorticity amplification at small scales, also explaining why the power law derived in Eq. (5) fails to capture the behavior of $R_{c}^{a}(\Omega)$ (in Fig. 3) for large $\Omega$. In contrast, for Burgers vortices, for which $R_{c}^{a}(\Omega)=R_{c}(\Omega)$, the stretching produced by local strain is always zero [13]; i.e., the self-attenuation mechanism is always absent.

The breakdown of scale invariance can further be shown by considering the critical scale $R_{c}^{P}=R_{c}^{P}(\Omega)$, defined by the condition that nonlocal enstrophy production recovers most of the total production (as shown in Fig. 6). Remarkably, we find that $R_{c}^{P}$ seemingly becomes constant at large $\Omega$, marking a critical scale below which the nonlocal effects do not penetrate and local dynamics dominate. A comparison with Fig. 3 shows that the value of $R_{c}^{P}$ and range of $\Omega$ where its constant are consistent with where $R_{c}^{a}$ deviates from $R_{c}$ - once again consistent with the onset of self-attenuation mechanism [15].

The breakdown of scale-invariance (self-similarity) of vortex stretching leads to some important consequences for turbulence theory and modeling. Prevalent intermittency theories postulate that gradient amplification and the resulting energy cascade is self-similar across scales, until regularized by viscosity. In fact, such an assumption is directly built into celebrated Kolmogorov's hypotheses and also multifractal and shell models [30]. However, current results point to an intricate role of nonlinearity, which acts in conjunction with viscosity to attenuate the most extreme events. This casts serious doubts on the dimensional estimate of the scale where viscous effects become prevalent, as used by phenomenological models. In fact, there is mounting evidence that such models are inadequate at characterizing extreme events, even 
at large Reynolds numbers [7,18,31]. A similar situation also applies to large-eddy simulation, where local dynamics are unresolved (by definition). The current results call for development of new models which can, for instance, appropriately capture the self-attenuation mechanism.

In conclusion, using state-of-the-art DNS, we have analyzed nonlocality of vorticity amplification by directly tackling the global Biot-Savart integral. We show that vorticity is predominantly amplified by nonlocal strain, with the underlying dynamics being linear. We identify the characteristic scale of nonlocality, which varies as a simple power law of vorticity magnitude. The nonlinear effects are captured by the remaining local strain, revealing that the nature of extreme events is fundamentally different due to the self-attenuation mechanism [15], ultimately leading to a breakdown of the observed power law and scale invariance of vortex-stretching mechanism. Further investigations are ongoing and are expected to provide essential ingredients for improved intermittency theories and turbulence models.

We gratefully acknowledge the Gauss Centre for Supercomputing e.V. for providing computing time on the supercomputers JUQUEEN and JUWELS at Jülich Supercomputing Centre (JSC), where the simulations reported in this paper were performed.
[1] D. R. Solli, C. Ropers, P. Koonath, and B. Jalali, Optical rogue waves, Nature (London) 450, 1054 (2007).

[2] S. Rahmstorf and D. Coumou, Increase of extreme events in a warming world, Proc. Natl. Acad. Sci. USA 108, 17905 (2011).

[3] T. P. Sapsis, Statistics of extreme events in fluid flows and waves, Annu. Rev. Fluid Mech. 53, 85 (2021).

[4] E. D. Siggia, Numerical study of small-scale intermittency in three-dimensional turbulence, J. Fluid Mech. 107, 375 (1981).

[5] J. Jimenez, A. A. Wray, P. G. Saffman, and R. S. Rogallo, The structure of intense vorticity in isotropic turbulence, J. Fluid Mech. 255, 65 (1993).

[6] T. Ishihara, T. Gotoh, and Y. Kaneda, Study of high-Reynoldsnumber isotropic turbulence by direct numerical simulations, Annu. Rev. Fluid Mech. 41, 165 (2009).

[7] D. Buaria, A. Pumir, E. Bodenschatz, and P. K. Yeung, Extreme velocity gradients in turbulent flows, New J. Phys. 21, 043004 (2019).

[8] H. Tennekes and J. L. Lumley, A First Course in Turbulence (MIT Press, Cambridge, UK, 1972).

[9] M. Carbone and A. D. Bragg, Is vortex stretching the main cause of the turbulent energy cascade? J. Fluid Mech. 883, R2 (2020).

[10] A. Pumir, A numerical study of pressure fluctuations in three-dimensional, incompressible, homogeneous, isotropic turbulence, Phys. Fluids 6, 2071 (1994).

[11] K. K. Nomura and G. K. Post, The structure and dynamics of vorticity and rate of strain in incompressible homogeneous turbulence, J. Fluid Mech. 377, 65 (1998).

[12] C. R. Doering, The 3D Navier-Stokes problem, Annu. Rev. Fluid Mech. 41, 109 (2009).

[13] P. E. Hamlington, J. Schumacher, and W. J. A. Dahm, Local and nonlocal strain rate fields and vorticity alignment in turbulent flows, Phys. Rev. E 77, 026303 (2008).

[14] P. E. Hamlington, J. Schumacher, and W. J. A. Dahm, Direct assessment of vorticity alignment with local and nonlocal strain rates in turbulent flows, Phys. Fluids 20, 111703 (2008).

[15] D. Buaria, A. Pumir, and E. Bodenschatz, Self-attenuation of extreme events in Navier-Stokes turbulence, Nat. Commun. 11, $5852(2020)$

[16] For a $N^{3}$ grid, the complexity of this integration scales as $\mathcal{O}\left(N^{6}\right)$, making it practically unfeasible.
[17] R. S. Rogallo, Numerical experiments in homogeneous turbulence, NASA Technical Memo 81315, 1981.

[18] D. Buaria and K. R. Sreenivasan, Dissipation range of the energy spectrum in high Reynolds number turbulence, Phys. Rev. Fluids 5, 092601(R) (2020).

[19] D. Buaria, E. Bodenschatz, and A. Pumir, Vortex stretching and enstrophy production in high Reynolds number turbulence, Phys. Rev. Fluids 5, 104602 (2020).

[20] D. Buaria, A. Pumir, and E. Bodenschatz, Generation of intense dissipation in high Reynolds number turbulence, arXiv:2107.01719, (2021).

[21] W. T. Ashurst, A. R. Kerstein, R. M. Kerr, and C. H. Gibson, Alignment of vorticity and scalar gradient with strain rate in simulated Navier-Stokes turbulence, Phys. Fluids 30, 2343 (1987).

[22] A. Tsinober, E. Kit, and T. Dracos, Experimental investigation of the field of velocity gradients in turbulent flows, J. Fluid Mech. 242, 169 (1992).

[23] B. Lüthi, A. Tsinober, and W. Kinzelbach, Lagrangian measurements of vorticity dynamics in turbulent flow, J. Fluid Mech. 528, 87 (2005).

[24] G. K. Batchelor, The Theory of Homogeneous Turbulence (Cambridge University Press, Cambridge, UK, 1953).

[25] J. Jimenez, Kinematic alignment effects in turbulent flows, Phys. Fluids 4, 652 (1992).

[26] H. K. Moffatt, S. Kida, and K. Ohkitani, Stretched vorticesthe sinews of turbulence: Large-Reynolds-number asymptotics, J. Fluid Mech. 259, 241 (1994).

[27] Note that at $R \rightarrow \infty$ the nonlocal strain vanishes, and hence all the alignments would just eventually approach $1 / 3$ corresponding to Gaussian background noise.

[28] See Supplemental Material at http://link.aps.org/supplemental/ 10.1103/PhysRevResearch.3.L042020 for the plot of strain conditioned on vorticity from Ref. [19], along with the dependence of $\gamma$ on $R_{\lambda}$.

[29] J. M. Burgers, A mathematical model illustrating the theory of turbulence, Adv. Appl. Mech. 1, 171 (1948).

[30] U. Frisch, Turbulence: The Legacy of Kolmogorov (Cambridge University Press, Cambridge, UK, 1995).

[31] G. Elsinga, T. Ishihara, and J. C. R. Hunt, Extreme dissipation and intermittency in turbulence at very high reynolds numbers, Proc. R. Soc. London A 476, 20200591 (2020). 